\title{
Frequency of occurrence and concreteness ratings of homograph meanings
}

\author{
KEITH A. WOLLEN, STEVEN D. COX, MARGARET M. COAHRAN, \\ DANIEL S. SHEA, and ROBERT F. KIRBY \\ Washington State University, Pullman, Washington 99164
}

\begin{abstract}
The purpose of this research was to establish norms for the relative frequency of use of the different meaning of common homographs (words that have one spelling but two or more distinctly different meanings) and to present ratings of the concreteness-abstractness of those meanings. A total of 108 subjects wrote a phrase or sentence using each of 120 homographs that were presented at a 15.5 -sec rate. For each homograph, norms are provided indicating the relative frequency with which each meaning was used by men and by women. In addition, four judges rated the concreteness-abstractness of each meaning. These ratings are also provided, as are the means of the overall concreteness for each homograph.
\end{abstract}

A homograph is a word that has a single spelling but two or more different meanings. Some people think of homographs as a small collection from the vast number of words that make up a lexicon. However, virtually any word has different meanings in different contexts and so could, in a sense, be viewed as a homograph. Thus, the study of homographs can be viewed as the study of a general characteristic of most words.

Perhaps for such reasons, homographs have become increasingly popular in psychological research in many different laboratories. Most of the uses center around the fact that homographs can be encoded in different ways. Because of this feature, homographs make excellent stimuli for experiments on set or priming (e.g., Cramer, 1968; Skanes \& Donderi, 1973), encoding specificity (e.g., Goldstein, Schmitt, \& Scheirer, 1978; Light \& Carter-Sobell, 1970; Winograd \& Conn, 1971), transfer (e.g., Hastroudi \& Johnson, 1976; Muller, Brown, \& Kausler, 1975), imagery (e.g., Begg \& Clark, 1975), frequency estimation (e.g., Geis \& Winograd, 1975; Rowe, 1973), clustering (e.g., Kausler \& Kamichoff, 1970), and cerebral laterality (Wollen, Cox, Coahran, \& Shea, Note 1).

To obtain homographs for research, many experimenters have had to scan dictionaries and thesauri. This is an extremely laborious and time-consuming method. Moreover, when completed, there are no normative data on such things as the relative frequencies of the various meanings. Thus, lists of homographs are clearly desirable.

There are a few existing lists, but most have weaknesses. For example, Fallows (1898) simply listed homographs and has no normative data whatsoever. Since the list is so old, many of the meanings do not apply today. Another source (Whitford, 1966) includes only those homographs that also have different pronunciations (e.g., refuse as a verb or a noun), and he provides no normative data.

Normative data are provided in the lists of Geis and
Winograd (1974) and Kausler and Kollasch (1970). However, these lists include only a small number of homographs (50 and 40, respectively) and hence are too restricted for many purposes. Also, only two meanings are usually included, even though many of the words have more meanings. Some meanings are omitted and others are combined (e.g., Kausler \& Kollasch combined meanings of "just" and "beautiful" for "fair," and "excellent" and "small" for "fine").

The most comprehensive list that also has normative data is provided by Cramer (1970). She used 100 homographs, which is a reasonably large number, and reported all responses made by her subjects. However, she provided no information concerning the relative number of males and females that participated in the research.

The Cramer (1970), Geis-Winograd (1974), and Kausler-Kollasch (1970) studies provided normative data on the frequencies of word associates to each homograph. One problem with the word-association approach is that it is sometimes impossible to tell exactly which meaning the subject encoded. For example, the stimulus "perch" had "sit" as a primary associate. Does "sit" refer to a noun (a place to sit) or to a verb (the act of sitting)? Another associate is "bird." Did the subject encode a noun (a bird perch) or a verb (a bird perching)? To preserve the precise meaning, we asked subjects to use each homograph in a phrase or sentence.

It is very likely that the encoding of a homograph will depend upon the preceding word or words. Previous norms have not exerted much control over this. Kausler and Kollasch (1970) used only two different random orders of the words, and Cramer (1970) had only one random order. Although Cramer rearranged the order of the pages, the words on a given page always appeared in the same sequence. Geis and Winograd (1974) recognized the problem and used four randomizations. We tried to gain still more generality by using 
six different randomizations, as well as by counterbalancing the pages on which the word appeared.

Previous researchers have used an unpaced procedure in which subjects respond to homographs in test booklets. Our experience has shown that subjects take much more care at first and then rush more and more as they tire of the task. To try to reduce this problem, we presented the homographs in a paced fashion so that the time spent on each word would be more nearly equalized.

\section{METHOD}

\begin{abstract}
Materials
The materials were 124 homographs that ranged from three to seven letters in length. The homographs were obtained by scanning the Macmillan Dictionary for Children (1975). This source was used because we wanted common words that virtually everyone would know. We excluded words if we felt that only one meaning was likely to be used. Each homograph was typed in capital letters and photographed. The resulting negatives were mounted onto slides, thereby producing a light image on a dark background. Of the homographs, 21 overlapped with the 40 used by Kausler and Kollasch (1970), 14 overlapped with the 50 used by Geis and Winograd (1974), and 32 overlapped with the 100 used by Cramer (1970). Well over half of the homographs reported herein (73 in all) have not appeared in any of the previous sets of norms. Primary emphasis was placed on selecting homographs that would be likely to have both concrete and abstract codings. Previous norms have not had this emphasis.
\end{abstract}

\section{Subjects}

The subjects were introductory psychology students whose participation partially fulfilled a course requirement. The subjects were run in six small groups ranging in size from 15 to 22 . Overall, there were 7 lefthanded and 40 righthanded men and 5 left handed and 56 righthanded women.

\section{Procedure}

The homographs were presented by means of a Kodak Carousel projector controlled by a Tally tape reader. Each slide was preceded by a $3,500-\mathrm{Hz}$ tone that sounded for $.5 \mathrm{sec}$ and served as a warning that another word was to appear. As the tone terminated, a homograph appeared and stayed on the screen for $15 \mathrm{sec}$. The offset of the homograph marked the onset of another tone that repeated the cycle. Subjects were told that the purpose of the experiment was to investigate how words are used in sentences and phrases. The subjects were instructed to look at the screen when they heard the tone and to write a phrase or sentence as quickly as they could when they saw the word. The subjects wrote their responses in test booklets that consisted of six pages with 20 blank lines per page.

The first four homographs were for practice only and so were not scored. At the end of the practice session, questions were answered. The 60 experimental homographs were shown, using the same procedure. After the first 64 words, there was a brief break while the experimenter changed the slide trays. Then the last 60 homographs were shown.

Each of the six groups of subjects had a completely different sequence of words. The 120 experimental homographs were divided into six sets of 20 words each. A Latin square was used to assign sets to sections within the list so that each set appeared first for one group of subjects, second for another, third for still another, and so on. The balancing was such that, across groups of subjects, each set : ppeared before every other set as well as after every other set. Within sets, the words were randomized separately for each group of subjects, with the restriction that obviously related words (e.g., toast and jam, spring and dart, permit and refuse) could not follow each other.

\section{RESULTS AND DISCUSSION}

The relative frequency of occurrence of each meaning of each homograph was determined separately for men and women. The resulting percentages are shown in Table 1 . The meanings are conveyed either by a brief definition or by using the word in a phrase, whichever seemed to communicate better. Table 1 does not include meanings used by only 1 or 2 of the 108 subjects. Also excluded are words for which all subjects used the same meaning. These words and the meanings used were "entrance" (an opening), "nap" (sleep), and "tense" (nervous). Thus, there are 117 homographs for which data are presented.

The percentages do not always add to 100 for several reasons. In many instances, there were meanings that were excluded because they were used by only one or two subjects. There was also a scattering of omissions, ambiguous meanings, and meanings inappropriate for the homograph. If such considerations are important for a given research project, it would be a simple matter to exclude words for which the sum of the percentages is appreciably below 100 . In general, there was a high correlation between the percentage use of specific meanings by men and women (Pearson $\mathrm{r}=.93$ ).

The comparability of our results to those of other investigators was also examined. This was accomplished by calculating the correlation coefficients between our norms and others, using whatever homographs were in common to the two sets of norms. Since some norms combined different meanings, meanings from our norms were similarly combined before calculating the correlations. In some cases it was not possible to know whether the combinations were the same as those used by other investigators, but the correlations should at least be approximations. Neither Cramer (1970) nor Geis and Winograd (1974) reported separate norms for males and females; hence, for comparisons with these data, it was necessary to average our values for males and females. The resulting correlations between our norms and others and the number of words in common were $(1) r=.73$ for Cramer, based on 32 common words; (2) $r=.95$ for Geis and Winograd, based on 14 common words; and (3) $I=.83$ (for males) and .87 (for females) for Kausler and Kollasch (1970), based on 21 common words. Thus, with the possible exception of the correlation with Cramer's norms, the correlations were fairly high.

Table 1 also includes a concreteness rating (designated by $C$ ) for each word meaning. These were determined by first making a list of all meanings for all homographs. Then four judges (the authors) ratad each meaning on concreteness. Concreteness was defined along the general lines of Spreen and Schulz (1966). 
Table 1

Percentage Frequency of Occurrence and Concreteness Ratings (C) for Men (M) and Women (W)

\begin{tabular}{|c|c|c|}
\hline & & Percentage \\
\hline Homograph & $\mathrm{C}$ & M $\quad$ W \\
\hline
\end{tabular}

ANNUAL (3.00-2.96)

yearly

a yearbook

BANK (3.96-3.97)

banks lend money

river bank

you can bank on it

BAR (4.914.88)

a tavern or counter

a bar of gold, etc.

bar exams

BAT (4.354.57)

an animal

baseball bat

to hit

Batman

"bat out of hell"

"bat an eyelash"

BEAR (2.49-2.60)

I cannot bear it

an animal

he is an old bear

BEING (2.24-2.10)

form of be

human being

BEND (3.21-3.08)

to bend over

to bend metal

$a$ bend in a river

to bend the rules

BLOCK (3.51-3.59)

a city block

to block traffic

a block of something

blockhead

to block progress

a mental block

BLUFF (2.47-2.39)

call his bluff

to bluff

a cliff

BLUNT (2.41-2.40)

a blunt person

a blunt object

BOIL (3.49-3.13)

to cook

a skin infection

BOOM (4.07-3.89)

a noise

a machine

baby boom, boom town

BROKE (2.48-2.52)

broke the vase

without money

broke a record, relationship

CAN (2.67-2.08)

to be able

a container

CHANGE (3.70-3.43)

coins

a difference

to change clothes, etc.

to change your mind

CHECK (4.17-4.00)

a bank draft

$\begin{array}{lll}2 & 60 & 66\end{array}$

$\begin{array}{lll}5 & 30 & 31\end{array}$

$\begin{array}{lll}4 & 85 & 87\end{array}$

$\begin{array}{lrr}5 & 4 & 7\end{array}$

$\begin{array}{lll}2 & 4 & 5\end{array}$

$\begin{array}{lll}5 & 77 & 90\end{array}$

$\begin{array}{rrr}5 & 13 & 3 \\ 1 & 2 & 3\end{array}$

$5 \quad 34 \quad 49$

$\begin{array}{lll}5 & 30 & 26\end{array}$

$\begin{array}{lll}3 & 19 & 10\end{array}$

$\begin{array}{lll}5 & 6 & 3\end{array}$

$\begin{array}{lll}1 & 4 & 2\end{array}$

123

$\begin{array}{lll}2 & 79 & 77\end{array}$

$\begin{array}{lll}5 & 13 & 18\end{array}$

$\begin{array}{lll}4 & 4 & 2\end{array}$

$\begin{array}{lll}1 & 57 & 59\end{array}$

$\begin{array}{lll}4 & 40 \quad 34\end{array}$

$\begin{array}{lll}3 & 28 & 43\end{array}$

$3 \quad 34 \quad 26$

$\begin{array}{lll}4 & 26 & 15\end{array}$

$\begin{array}{lll}2 & 6 & 8\end{array}$

$\begin{array}{lll}5 & 26 & 33\end{array}$

$4 \quad 15 \quad 21$

167

$\begin{array}{lll}2 & 8 & 2\end{array}$

$\begin{array}{lll}1 & 2 & 8\end{array}$

$\begin{array}{lll}2 & 53 & 44\end{array}$

$\begin{array}{lll}2 & 28 & 36\end{array}$

$\begin{array}{lll}5 & 15 & 12\end{array}$

$\begin{array}{lll}2 & 55 & 59\end{array}$

$\begin{array}{lll}3 & 38 & 39\end{array}$

$3 \quad 67 \quad 90$

$5 \quad 226$

$\begin{array}{rrr}5 & 6 & 3\end{array}$

$\begin{array}{lll}3 & 47 & 52\end{array}$

$245 \quad 41$

$\begin{array}{lll}2 & 6 & 7\end{array}$

$\begin{array}{lll}1 & 53 & 70\end{array}$

$\begin{array}{lll}5 & 38 & 26\end{array}$

$\begin{array}{lll}5 & 53 & 43\end{array}$

$221 \quad 23$

$\begin{array}{lll}3 & 17 & 25\end{array}$

$\begin{array}{lll}1 & 8 & 10\end{array}$

$\begin{array}{lll}5 & 62 & 49\end{array}$ $\begin{array}{lll}3 & 28 & 23\end{array}$

$\begin{array}{lll}4 & 81 & 87\end{array}$
Table 1 Continued

\begin{tabular}{lrrr}
\hline & \multicolumn{3}{c}{ Percentage } \\
Homograph & C & M & W \\
\hline & 2 & 15 & 28 \\
to check a test & 5 & 4 & 16 \\
check book, account & 2 & 9 & 3 \\
check that out & 3 & 2 & 3
\end{tabular}

CLIP (3.81-3.95)

a fastener

to cut

to fasten

to hit

a hit in football

a clip-on earring

COLD (3.88-3.86)

cold in temperature

a disease

unfriendly

COMPACT (3.33-3.59)

car

small, densely packed

cosmetic kit

to compact

CONSOLE (3.40-2.36)

to comfort

a cabinet

COUNT (3.18-2.99)

to count objects

count on us

Count Dracula, etc.

the final count

CRANE (4.85-4.93)

a machine

a bird

crane your neck

CUE (4.31 -4.04)

a signal

pool cue

cue ball or cards

CURB (4.50-4.55)

curb of a street

to curb appetite, impulse

to curb a dog

DART (4.674.48)

a small missle

to move quickly

a game

Dodge Dart

DESERT (4.87-4.75)

arid region

a dessert

to abandon

DIGEST (3.59-2.93)

a magazine or diary

to digest food

to digest information

DOVE (4.10-4.29)

a bird

dove into the pool

a symbol of peace

political dove

DOWN (3.20-2.96)

a spatial location

feathers

downtown, down the road

he is down (emotionally)

prices, grades go down

DRY (3.46-3.48)

not wet

to dry off $\begin{array}{lll}5 & 34 & 41\end{array}$

$3 \quad 34 \quad 33$

$\begin{array}{lll}3 & 9 & 10\end{array}$

$3 \quad 9 \quad 3$

$\begin{array}{lll}4 & 6 & 3 \\ 4 & 2 & 5\end{array}$

$\begin{array}{lll}4 & 79 & 74\end{array}$

$\begin{array}{lll}4 & 15 & 18\end{array}$

267

$\begin{array}{lll}4 & 57 & 56\end{array}$

$230 \quad 25$

$\begin{array}{lll}5 & 2 & 13\end{array}$

362

$\begin{array}{lll}2 & 26 & 72\end{array}$

$4 \quad 60 \quad 16$

$3 \quad 46 \quad 59$

$2 \quad 11 \quad 21$

$\begin{array}{lll}5 & 13 & 10\end{array}$

$\begin{array}{lll}3 & 15 & 7\end{array}$

$\begin{array}{lll}5 & 53 & 54\end{array}$

$5 \quad 23 \quad 26$

363

$3 \quad 34 \quad 44$

$\begin{array}{lll}5 & 38 & 18\end{array}$

$\begin{array}{lll}5 & 26 & 30\end{array}$

$\begin{array}{lll}5 & 79 & 84\end{array}$

$2 \quad 11 \quad 13$

$\begin{array}{lll}3 & 78 & 83\end{array}$

$\begin{array}{lll}5 & 64 & 59\end{array}$

$3 \quad 13 \quad 18$

$4 \quad 6 \quad 16$

5157

$\begin{array}{lll}5 & 55 & 59\end{array}$

53630

248

$5 \quad 51 \quad 31$

$2 \quad 45 \quad 59$

2010

$\begin{array}{lll}5 & 53 & 61\end{array}$

$3 \quad 34 \quad 13$

3215

243

$\begin{array}{lll}3 & 38 & 52\end{array}$

$5 \quad 23 \quad 13$

2620

$211 \quad 8$

$211 \quad 2$

$\begin{array}{lll}4 & 53 & 61\end{array}$
$3 \quad 21 \quad 26$ 
Table 1 Continued

\begin{tabular}{|c|c|c|c|}
\hline \multirow{2}{*}{ Homograph } & \multicolumn{3}{|c|}{ Percentage } \\
\hline & C & $\mathrm{M}$ & W \\
\hline thirsty & 2 & 11 & 0 \\
\hline dry throat or lips & 3 & 6 & 3 \\
\hline dry humor & 1 & 0 & 7 \\
\hline \multicolumn{4}{|l|}{ DUCK $(4.56-4.62)$} \\
\hline an animal & 5 & 64 & 75 \\
\hline to duck down & 3 & 21 & 18 \\
\hline duck pond & 5 & 11 & 3 \\
\hline \multicolumn{4}{|l|}{ FAIR $(3.61-2.97)$} \\
\hline county fair & 5 & 51 & 38 \\
\hline equitable & 1 & 30 & 46 \\
\hline fair skies or skin & 4 & 19 & 13 \\
\hline \multicolumn{4}{|l|}{ FAST $(2.00-2.00)$} \\
\hline quick, quickly & 2 & 81 & 80 \\
\hline to go without food & 2 & 6 & 8 \\
\hline promiscuous & 2 & 4 & 5 \\
\hline \multicolumn{4}{|l|}{ FELT (2.67-2.59) } \\
\hline I felt ill & 2 & 47 & 57 \\
\hline I felt the texture & 3 & 34 & 25 \\
\hline a cloth & 5 & 13 & 13 \\
\hline I felt it was wrong & 1 & 6 & 5 \\
\hline \multicolumn{4}{|l|}{ FIGURE $(3.36-3.20)$} \\
\hline a shape (person or thing) & 4 & 62 & 48 \\
\hline to consider & 2 & 32 & 39 \\
\hline a number & 4 & 2 & 7 \\
\hline figure skater & 4 & 4 & 3 \\
\hline \multicolumn{4}{|l|}{ FILE (3.65-3.56) } \\
\hline to arrange & 3 & 30 & 34 \\
\hline a container & 5 & 13 & 18 \\
\hline to scrape & 3 & 13 & 15 \\
\hline a set of records & 4 & 17 & 8 \\
\hline a tool & 5 & 8 & 5 \\
\hline to file for divorce & 2 & 2 & 3 \\
\hline on fille & 2 & 2 & 3 \\
\hline \multicolumn{4}{|l|}{ FINE $(2.05-1.89)$} \\
\hline I am (feeling) fine & 2 & 32 & 30 \\
\hline library fine, etc. & 2 & 19 & 20 \\
\hline fine time, he did fine & 1 & 17 & 21 \\
\hline fine house & 2 & 15 & 21 \\
\hline fine sandpaper, wire & 4 & 11 & 5 \\
\hline \multicolumn{4}{|l|}{ FLAT (3.64-3.46) } \\
\hline flat surface & 3 & 64 & 70 \\
\hline flat (tire) & 5 & 28 & 18 \\
\hline an apartment & 5 & 2 & 3 \\
\hline \multicolumn{4}{|l|}{ FLY (3.94-3.72) } \\
\hline to fly in air & 3 & 49 & 54 \\
\hline an insect & 5 & 43 & 34 \\
\hline pants placket & 5 & 6 & 3 \\
\hline fly-by-night & 1 & 2 & 3 \\
\hline \multicolumn{4}{|l|}{ FOIL (4.27-4.79) } \\
\hline thin metal & 5 & 66 & 90 \\
\hline to thwart & 2 & 23 & 7 \\
\hline fencing foil & 5 & 6 & 2 \\
\hline \multicolumn{4}{|l|}{ FORCE (2.59-3.00) } \\
\hline to force a door or person & 3 & 26 & 33 \\
\hline strength & 3 & 17 & 34 \\
\hline "The Force" (Star Wars) & 1 & 30 & 8 \\
\hline police, air, etc., force & 4 & 21 & 16 \\
\hline \multicolumn{4}{|l|}{ FRESH $(2.00-2.00)$} \\
\hline uses implying newness or purity & 2 & 79 & 79 \\
\hline flirtations & 2 & 19 & 15 \\
\hline GRATE (3.44-3.20) & & & \\
\hline to grate cheese & 3 & 53 & 54 \\
\hline a metal grille & 5 & 23 & 18 \\
\hline to grate on nerves & 2 & 9 & 18 \\
\hline
\end{tabular}

Table 1 Continued

Homograph

Percentage

GROSS (1.53-1.62)

a gross person

gross weight, profit, atc.

12 dozen

goss understatement

GROUND (4.78-4.73)

earth

groundhog

ground floor

chopped

she ground corn

HAMPER (3.88-4.29)

a container

to hinder

HEAD (3.49-3.72)

head of a body, bed, etc.

a good head (intelligent)

a leader

to head south

a brand name

sexual slang

drug slang

ahead

a big head (inflated ego)

HIDE (3.29-3.21)

to conceal

hide and seek (game)

animal skin

hideaway

HOLD (2.46-2.62)

to grip an object or person

to wait or to keep (hold it, hold still)

put on hold

a grip

to have an emotional hold

HOST (3.84-3.94)

a host of a party

to host a party

a multitude

HUSKY (4.37-4.15)

husky body

a dog

(a school mascot)

husky voice

INTEREST (1.85-1.89)

to have interest in

interest on a loan

INVALID (2.39-2.96)

not valid

a sick person

IRON (3.64-4.16)

a metal

to iron a shirt

an appliance

iron pills

muscles of iron

JAM (4.41 4.28)

a food

a difficult situation

jam session

traffic jam

jammed door, etc.

to jam into

KIND (1.58-1.79)
C $\mathrm{M}$ W

$\begin{array}{lll}2 & 38 & 59\end{array}$

$140 \quad 33$

$211 \quad 0$

14

$5 \quad 74 \quad 70$

$\begin{array}{lll}5 & 4 & 13\end{array}$

$\begin{array}{lll}4 & 4 & 7\end{array}$

$\begin{array}{lll}3 & 6 & 3 \\ 3 & 2 & 7\end{array}$

$\begin{array}{lll}5 & 74\end{array}$

$234 \quad 23$

$5 \quad 32 \quad 43$

$119 \quad 10$

$\begin{array}{lll}4 & 4 & 16\end{array}$

$14 \quad 10$

$5 \quad 11 \quad 3$

$\begin{array}{lll}3 & 9 & 0\end{array}$

4442

$3 \quad 4 \quad 2$

$1 \quad 23$

$\begin{array}{lll}5 & 13 & 8\end{array}$

$\begin{array}{lll}4 & 2 & 5\end{array}$

$\begin{array}{lll}3 & 36 & 48\end{array}$

$234 \quad 33$

$213 \quad 5$

$\begin{array}{lll}4 & 2 & 5\end{array}$

$\begin{array}{lll}4 & 2 & 3\end{array}$

$4 \quad 94 \quad 92$

233

140

$\begin{array}{lll}4 & 30 & 46\end{array}$

$\begin{array}{lll}5 & 36 & 21\end{array}$

$\begin{array}{lll}4 & 32 & 18\end{array}$

307

$\begin{array}{lll}2 & 83 & 87\end{array}$

$115 \quad 11$

$1 \quad 64 \quad 51$

$\begin{array}{lll}5 & 34 & 49\end{array}$

$\begin{array}{lll}5 & 26 & 57\end{array}$

$3 \quad 30 \quad 11$

$3 \quad 13 \quad 25$

$315 \quad 5$

443

$\begin{array}{lll}5 & 68 & 64\end{array}$

$\begin{array}{lll}2 & 8 & 12\end{array}$

$3 \quad 11 \quad 5$

$\begin{array}{lll}4 & 6 & 7\end{array}$

305 $\begin{array}{lll}3 & 68 & 54\end{array}$

$3 \quad 15 \quad 33$ 
Table 1 Continued

\begin{tabular}{|c|c|c|c|}
\hline \multirow{2}{*}{ Homograph } & \multirow[b]{2}{*}{$\mathrm{C}$} & \multicolumn{2}{|c|}{ Percentage } \\
\hline & & M & W \\
\hline a kind person & 2 & 57 & 79 \\
\hline a kind of car & 1 & 38 & 16 \\
\hline I kind of like her & 1 & 4 & 5 \\
\hline \multicolumn{4}{|l|}{ LASH $(3.70-3.56)$} \\
\hline eyelash & 5 & 23 & 28 \\
\hline to lash out & 2 & 19 & 31 \\
\hline give him 30 lashes & 4 & 21 & 15 \\
\hline to tie & 3 & 15 & 8 \\
\hline to whip & 3 & 6 & 5 \\
\hline a whipping tool & 5 & 4 & 3 \\
\hline whiplash & 5 & 4 & 3 \\
\hline \multicolumn{4}{|l|}{ LEAD (3.65-3.55) } \\
\hline to lead a horse, etc. & 3 & 17 & 43 \\
\hline a metal & 5 & 43 & 13 \\
\hline pencil lead & 5 & 8 & 23 \\
\hline "get the lead out" & 1 & 19 & 10 \\
\hline $\begin{array}{l}\text { take the lead, etc. } \\
\text { LIGHT }(4.80-4.51)\end{array}$ & 3 & 11 & 5 \\
\hline $\begin{array}{l}\text { LIGHT (4.80-4.51) } \\
\text { light bulb, switch, etc. }\end{array}$ & LIGHT $(4.80-4.51)$ & 40 & 38 \\
\hline $\begin{array}{l}\text { light bulb, switch, etc. } \\
\text { it is light out }\end{array}$ & 4 & 40 & 34 \\
\hline $\begin{array}{l}\text { it is light out } \\
\text { metaphorical uses (e.g., see the light) }\end{array}$ & 1 & 8 & 11 \\
\hline $\begin{array}{l}\text { metaphorical uses (e.g., see the light) } \\
\text { to light a fire }\end{array}$ & 3 & 2 & 10 \\
\hline light in weight & 2 & 4 & 2 \\
\hline \multicolumn{4}{|l|}{ LIKE $(2.00-2.00)$} \\
\hline I like cake & 2 & 89 & 93 \\
\hline $\begin{array}{l}\text { similar to, she looks like you, etc, } \\
\text { LOAF }(3.77-3.76)\end{array}$ & 2 & 11 & 7 \\
\hline bread, meat loaf & 4 & 77 & 74 \\
\hline to loaf & 3 & 23 & 23 \\
\hline \multicolumn{4}{|l|}{ LOUNGE (4.58-4.48) } \\
\hline a room & 5 & 74 & 69 \\
\hline to be idle & 3 & 21 & 26 \\
\hline lounge chair & 5 & 4 & 5 \\
\hline \multicolumn{4}{|l|}{ MIGHT $(1.56-1.22)$} \\
\hline maybe & 1 & 72 & 87 \\
\hline strength & 3 & 28 & 11 \\
\hline \multicolumn{4}{|l|}{ MINE (2.08-1.81) } \\
\hline belonging to me & 1 & 68 & 75 \\
\hline coal, etc., mine & 5 & 19 & 16 \\
\hline explosive device & 5 & 6 & 3 \\
\hline \multicolumn{4}{|l|}{ MOLD (4.49-4.47) } \\
\hline mold on cheese & 5 & 68 & 69 \\
\hline jello, etc., mold & 4 & 17 & 16 \\
\hline to mold clay & 3 & 11 & 8 \\
\hline to mold personality & 2 & 4 & 7 \\
\hline \multicolumn{4}{|l|}{ MOLE $(4.87-4.78)$} \\
\hline an animal & 5 & 49 & 43 \\
\hline a body spot & 5 & 40 & 44 \\
\hline chemical unit & 2 & 4 & 7 \\
\hline \multicolumn{4}{|l|}{ NET $(4.07-3.95)$} \\
\hline fish, tennis, etc., net & 5 & 72 & 72 \\
\hline net profit, weight & 1 & 19 & 25 \\
\hline to net a fish & 3 & 8 & 2 \\
\hline \multicolumn{4}{|l|}{ NOVEL $(1.76-1.72)$} \\
\hline new & 1 & 81 & 82 \\
\hline a book & 5 & 19 & 18 \\
\hline \multicolumn{4}{|l|}{ OBJECT $(2.53-2.23)$} \\
\hline to object & 2 & 34 & 33 \\
\hline some material thing & 4 & 36 & 28 \\
\hline the purpose & 1 & 23 & 34 \\
\hline ODD $(1.00-1.00)$ & & & \\
\hline unusual & 1 & 79 & 93 \\
\hline not even & 1 & 11 & 3 \\
\hline ORGAN $(4.81-4.84)$ & & & \\
\hline a musical instrument & 5 & 81 & 82 \\
\hline
\end{tabular}

Table 1 Continued

\begin{tabular}{|c|c|c|c|}
\hline \multirow[b]{2}{*}{ Homograph } & \multirow[b]{2}{*}{$\mathrm{C}$} & \multicolumn{2}{|c|}{ Percentage } \\
\hline & & $\mathbf{M}$ & $\mathbf{W}$ \\
\hline \multicolumn{4}{|l|}{$\begin{array}{l}\text { a part of the body } \\
\text { PAGE }(4.82-4.76)\end{array}$} \\
\hline a book page & 5 & 79 & 84 \\
\hline a messenger & 4 & 6 & 10 \\
\hline to call someone & 3 & 6 & 7 \\
\hline a person's name & 5 & 8 & 0 \\
\hline \multicolumn{4}{|l|}{ PEER (3.45-3.40) } \\
\hline an equal & 4 & 68 & 66 \\
\hline to look & 2 & 26 & 28 \\
\hline \multicolumn{4}{|l|}{ PELT $(4.72-4.63)$} \\
\hline animal skin & 5 & 79 & 67 \\
\hline to strike & 3 & 13 & 15 \\
\hline \multicolumn{4}{|l|}{ PERCH (4.17-3.91) } \\
\hline a fish & 5 & 47 & 25 \\
\hline to perch & 3 & 30 & 34 \\
\hline a resting place & 4 & 21 & 36 \\
\hline \multicolumn{4}{|l|}{ PERFECT (1.02-1.07) } \\
\hline it is perfect & 1 & 98 & 93 \\
\hline to perfect the process & 2 & 2 & 7 \\
\hline \multicolumn{4}{|l|}{ PERMIT $(3.32-3.40)$} \\
\hline a document & 4 & 66 & 70 \\
\hline to allow & 2 & 34 & 30 \\
\hline \multicolumn{4}{|l|}{ PICK $(2.93-2.93)$} \\
\hline to pick up (grasp objects) & 3 & 38 & 36 \\
\hline to choose & 2 & 23 & 26 \\
\hline a tool & 5 & 13 & 15 \\
\hline to pick nose, teeth & 3 & 13 & 8 \\
\hline to tease & 2 & 6 & 3 \\
\hline take your pick & 2 & 4 & 3 \\
\hline pick us up & 2 & 0 & 5 \\
\hline \multicolumn{4}{|l|}{ PIT $(4.32-4.18)$} \\
\hline a hole & 5 & 45 & 33 \\
\hline fruit pit & 5 & 9 & 30 \\
\hline this is the pits & 2 & 13 & 23 \\
\hline this room is a pit & 4 & 21 & 7 \\
\hline \multicolumn{4}{|l|}{ PLANE $(4.35-4.87)$} \\
\hline airplane & 5 & 77 & 92 \\
\hline plane geometry & 1 & 15 & 3 \\
\hline \multicolumn{4}{|l|}{ PLOT $(2.27-2.14)$} \\
\hline a plan & 2 & 79 & 82 \\
\hline a plot of land & 4 & 13 & 7 \\
\hline to plan & 2 & 6 & 8 \\
\hline \multicolumn{4}{|l|}{ PRESENT (2.83-2.85) } \\
\hline a gift & 4 & 43 & 43 \\
\hline to be present at a meeting & 2 & 26 & 18 \\
\hline now, at this time & 1 & 17 & 20 \\
\hline to present an award or speech & 3 & 6 & 16 \\
\hline to introduce someone & 3 & 8 & 3 \\
\hline \multicolumn{4}{|l|}{ PRESS $(3.40-3.49)$} \\
\hline newspeople & 4 & 28 & 31 \\
\hline to push & 3 & 23 & 23 \\
\hline to iron clothes & 3 & 19 & 20 \\
\hline a machine & 5 & 8 & 12 \\
\hline the newspaper process & 3 & 11 & 5 \\
\hline to press for an answer & 2 & 2 & 5 \\
\hline press your luck & 2 & 4 & 2 \\
\hline PRUNE (4.434.77) & & & \\
\hline a fruit & 5 & 62 & 85 \\
\hline to prune shrubs & 3 & 19 & 8 \\
\hline "old prune," "prune face" & 4 & 19 & 7 \\
\hline PUNCH $(4.32-4.22)$ & & & \\
\hline a drink & 5 & 60 & 57 \\
\hline to hit & 3 & 34 & 38 \\
\hline Punch and Judy & 5 & 6 & 3 \\
\hline
\end{tabular}


Table 1 Continued

\begin{tabular}{|c|c|c|c|}
\hline \multirow{2}{*}{ Homograph } & \multirow[b]{2}{*}{$\mathrm{C}$} & \multicolumn{2}{|c|}{ Percentage } \\
\hline & & M & W \\
\hline part of the eye & 5 & 53 & 69 \\
\hline a student & 5 & 47 & 31 \\
\hline \multicolumn{4}{|l|}{ RACKET $(4.15-4.28)$} \\
\hline tennis racket & 5 & 53 & 48 \\
\hline racquetball & 4 & 23 & 28 \\
\hline a scheme & 1 & 13 & 7 \\
\hline noise & 4 & 6 & 13 \\
\hline \multicolumn{4}{|l|}{ RASH (3.83-3.96) } \\
\hline a skin rash & 5 & 68 & 74 \\
\hline a rash decision & 1 & 26 & 23 \\
\hline \multicolumn{4}{|l|}{ REFUSE $(2.26-2.10)$} \\
\hline to reject & 2 & 87 & 95 \\
\hline trash & 4 & 13 & 5 \\
\hline \multicolumn{4}{|l|}{ ROCK $(4.334 .56)$} \\
\hline a stone & 5 & 47 & 77 \\
\hline music & 4 & 32 & 28 \\
\hline to sway & 3 & 6 & 5 \\
\hline do not rock the boat & 1 & 4 & 3 \\
\hline \multicolumn{4}{|l|}{ ROOT (3.41-3.36) } \\
\hline part of a plant & 5 & 47 & 48 \\
\hline root of the problem & 1 & 27 & 34 \\
\hline root beer & 5 & 4 & 8 \\
\hline to dig & 3 & 9 & 0 \\
\hline family history & 2 & 2 & 5 \\
\hline square root & 1 & 4 & 2 \\
\hline \multicolumn{4}{|l|}{ ROSE (4.91 -4.83) } \\
\hline a flower & 5 & 79 & 82 \\
\hline I rose from the chair & 3 & 4 & 8 \\
\hline a girl's name & 5 & 4 & 5 \\
\hline \multicolumn{4}{|l|}{ RUNG (3.65-3.34) } \\
\hline bell has rung & 3 & 32 & 48 \\
\hline "wrung" & 3 & 34 & 31 \\
\hline ladder rung & 5 & 32 & 16 \\
\hline \multicolumn{4}{|l|}{ SAW $(3.16-3.01)$} \\
\hline I saw them & 2 & 49 & 61 \\
\hline a cutting tool & 5 & 32 & 31 \\
\hline to cut & 3 & 15 & 7 \\
\hline \multicolumn{4}{|l|}{ SEAL $(4.264 .22)$} \\
\hline an animal & 5 & 43 & 48 \\
\hline a seal (Easter, jar, etc.) & 5 & 23 & 16 \\
\hline to seal something & 3 & 17 & 20 \\
\hline my seal of approval & 2 & 11 & 7 \\
\hline seal with a kiss & 3 & 2 & 8 \\
\hline \multicolumn{4}{|l|}{ SENSE (1.09-1.19) } \\
\hline to make sense & 1 & 36 & 28 \\
\hline sense of humor, direction, etc. & 1 & 15 & 23 \\
\hline common sense & 1 & 23 & 13 \\
\hline sixth sense & 1 & 17 & 15 \\
\hline to sense, use the senses & 2 & 9 & 18 \\
\hline \multicolumn{4}{|l|}{ SHED $(4.15-4.06)$} \\
\hline a building & 5 & 55 & 52 \\
\hline to shed hair, clothes, tears, etc. & 3 & 41 & 46 \\
\hline \multicolumn{4}{|l|}{ SOLE $(3.95-3.95)$} \\
\hline shoe sole & 5 & 64 & 52 \\
\hline only & 1 & 15 & 16 \\
\hline filet of sole & 5 & 9 & 21 \\
\hline "soul" & 1 & 11 & 10 \\
\hline SOW (4.25-4.13) & & & \\
\hline female hog & 5 & 53 & 53 \\
\hline to sow seeds & 3 & 32 & 41 \\
\hline SPRING $(2.56-2.23)$ & & & \\
\hline season of the year & 2 & 77 & 85 \\
\hline
\end{tabular}

Table 1 Continued

\begin{tabular}{lccc} 
& \multicolumn{3}{c}{ Percentage } \\
Homograph & C & M & W \\
\hline to move quickly & 3 & 4 & 8 \\
water & 5 & 6 & 5 \\
bed, etc., spring & 5 & 11 & 0 \\
SQUASH (4.48-4.42) & & & \\
a vegetable & 5 & 62 & 62 \\
a game & 4 & 21 & 18 \\
to squash & 3 & 15 & 20 \\
STABLE (3.43-3.76) & & & \\
horse's stable & 5 & 53 & 66 \\
emotionally balanced & 1 & 19 & 20 \\
condition of health & 2 & 15 & 10 \\
mechanically balanced , unchanging & 2 & 11 & 5
\end{tabular}

STEER (4.31-3.81)

an animal

to steer a car, etc.

to steer clear of

STICK (3.56-3.48)

a piece of wood

to adhere

stick to it

stick it to him, etc.

"stick in the mud"

"get on the stick"

stick shift

STRESS (2.22-2.02)

mental stress

mechanical stress

to stress a point

STRIP (3.42-3.44)

to remove something

a strip of bacon, wood, etc.

air strip, Las Vegas strip, etc.

strip poker of strip tease

SUIT (4.45-4.41)

clothing

law suit

to please

SWALLOW (3.30-3.31)

to swallow food

a bird

to believe

TEAR (4.064.13)

tear drops

to rip

a rip

TEND (1.35-1.26)

to have a tendency to

to take care of

to pay attention to

TIRE (4.51 4.49)

a tire for a car, etc.

to get tired

TOAST $(4.54-4.53)$

a form of bread

a proposed drink

to toast bread

WAKE (3.56-3.33)

wake up from sleep

boat's wake

funeral wake

WATCH (3.59-3.55)

a timepiece

to observe

$64 \quad 46$

$3 \quad 30 \quad 41$

$2 \quad 2 \quad 12$

$5 \quad 40 \quad 46$

$\begin{array}{lll}3 & 19 & 8\end{array}$

$\begin{array}{lll}2 & 6 & 18\end{array}$

197

$\begin{array}{lll}1 & 2 & 10\end{array}$

160

54

$\begin{array}{lll}2 & 70 & 89\end{array}$

$\begin{array}{lll}3 & 21 & 2\end{array}$

$\begin{array}{lll}2 & 4 & 8\end{array}$

$\begin{array}{lll}3 & 38 & 39\end{array}$

$4 \quad 21 \quad 30$

$4 \quad 19 \quad 13$

$\begin{array}{lll}3 & 17 & 16\end{array}$

$83 \quad 82$

$213 \quad 3$

1412

$\begin{array}{lll}3 & 70 & 77\end{array}$

$\begin{array}{lll}5 & 17 & 18\end{array}$

$2 \quad 6 \quad 5$

$5 \quad 49 \quad 51$

$\begin{array}{lll}3 & 43 & 38\end{array}$

$4 \quad 6 \quad 11$

$\begin{array}{lll}64 & 74\end{array}$

$\begin{array}{lll}2 & 17 & 16\end{array}$

$\begin{array}{lll}2 & 17 & 10\end{array}$

$\begin{array}{lll}5 & 77 & 79\end{array}$

$\begin{array}{lll}2 & 15 & 16\end{array}$

$\begin{array}{lll}5 & 77 & 74\end{array}$

$\begin{array}{rrr}3 & 17 & 21 \\ 3 & 6 & 2\end{array}$

$\begin{array}{lll}3 & 57 & 82\end{array}$

$\begin{array}{lll}5 & 17 & 15\end{array}$

$\begin{array}{lll}4 & 17 \quad 3\end{array}$

$\begin{array}{lll}5 & 53 & 51\end{array}$

$2 \quad 28 \quad 36$ 
Table 1 Continued

\begin{tabular}{lcrc}
\hline & \multicolumn{3}{c}{ Percentage } \\
Homograph & C & M & W \\
\hline watch out & 2 & 19 & 12 \\
WELL (3.49-3.04) & & & \\
oil, water well & 5 & 54 & 41 \\
I am well & 2 & 23 & 38 \\
he did well & 1 & 8 & 12 \\
oh well, etc. & 1 & 11 & 8 \\
WILL (1.71-2.09) & & & \\
I will go & 1 & 55 & 56
\end{tabular}

Table 1 Continued

\begin{tabular}{lcrr}
\hline & \multicolumn{3}{c}{ Percentage } \\
Homograph & C & M & W \\
\hline a legal document & 4 & 23 & 36 \\
her will, will power & 1 & 19 & 7 \\
WOUND (4.06-4.40) & & & \\
a physical injury & 5 & 55 & 72 \\
past of "to wind" & 3 & 21 & 10 \\
to injure physically & 3 & 8 & 7 \\
all wound up (tense) & 2 & 8 & 5 \\
to hurt someone's feelings & 2 & 2 & 3
\end{tabular}

Meanings that referred to material objects that could be experienced directly by the senses were assigned a rating of 5 (concrete), and meanings that could not be experienced directly by the senses were assigned a rating of 1 (abstract).

The judges first agreed on one or two exemplars of each of the five possible ratings. Then each judge rated a particular meaning. If three of the four judges agreed, the meaning was added to the list and another meaning was evaluated. Thus, each new meaning was compared with the meanings that had already been rated; this technique was used to reduce the number of internal inconsistencies within the ratings. If there was not agreement among three of the four judges, a fifth judge (an undergraduate student) resolved the split.

It is not possible to describe exactly the bases for assigning words to the five rating categories, but the types of words in each category did show some general trends. Meanings given a rating of 5 were generally specific objects, typically nouns, such as "tire" (vehicle), "toast" (food), "fly" (insect), and "lead" (metal). Ratings of 4 were more general things, such as "object" (some material thing), "present" (gift), and "force" (police). Ratings of 3 were primarily verbs depicting specific actions, such as "punch" (to hit), "saw" (to cut), and "hold" (to grip). Ratings of 2 consisted of verbs describing nonspecific actions, such as "hamper" (to hinder), "object" (to oppose), and "plot" (to plan). Finally, ratings of 1 consisted of various parts of speech, such as "can" (to be able), "net" (profit), "odd" (unusual), "object" (purpose), and "well" (as in "oh, well'). Over all 117 words, the number of meanings with ratings of $1,2,3,4$, and 5 were $58,93,93,56$, and 115 , respectively.

The numbers in parentheses beside each word in Table 1 are the means of the concreteness ratings for men (the first number) and women (the second number). These means were based only on the data shown in Table 1; in other words, meanings not used by at least three subjects (men and women combined) were excluded. The mean of these means for all words was 3.48 for men and 3.43 for women, a difference that did not reach statistical significance $[F(1,232)<1.00]$.
Thus, although the mean concreteness was higher for men on some words and higher for women on others, the overall level of concreteness was about the same for both sexes. The utility of the concreteness ratings was demonstrated by Wollen et al. (Note 1), who used 64 of the homographs. In that experiment, words presented to the left hemisphere were coded more concretely than words presented to the right hemisphere for men but not for women.

An internal analysis of the concreteness ratings revealed that the primary (most frequent) coding tended to be more concrete than the secondary (next most frequent) coding. For males, the means for the primary and secondary responses of all 117 homographs were 3.70 and 3.19 , respectively $(t=2.74)$. For females, the corresponding values were 3.60 and $3.16(t=2.40)$. Hence, it appears that, when there is a choice, subjects will respond more often with a concrete coding than with an abstract one.

\section{REFERENCE NOTE}

1. Wollen, K. A., Cox, S. D., Coahran, M. M., \& Shea, D. S. Homograph coding and cerebral laterality. Paper presented at the meeting of the Psychonomic Society, Phoenix, November 1979.

\section{REFERENCES}

BegG, I., \& Clark, J. M. Contextual imagery in meaning and memory. Memory \& Cognition, 1975, 3, 117-122.

Cramer, P. Mediated priming of polysemous stimuli. Journal of Experimental Psychology, 1968, 78, 137-144.

Cramer, P. A study of homographs. In L. Postman \& G. Keppel (Eds.), Norms of word association. New York: Academic Press, 1970.

Fallows, A. M. A complete dictionary of synonyms and antonyms. New York: Revell, 1898.

Geis, M. F., \& Winograd, E. Norms of semantic encoding variability for fifty homographs. Bulletin of the Psychonomic Society, 1974, 3, 429-431.

Geis, M. F., \& Winograd, E. Semantic encoding and judgments of background and situational frequency for homographs. Journal of Experimental Psychology: Human Learning and Memory, 1975, 1, 385-392.

Goldstein, E., Schmitt, J. C., \& Scheirer, C. J. Semantic effects in encoding specificity: A levels of processing approach. Memory \& Cognition, 1978, 6, 13-19.

Hashtroudi, S., \& Johnson, M. K. Transfer and forgetting: 
Interpretive shifts and stimulus reinstatement. Journal of Experimental Psychology: Human Learning and Memory, 1976, 2, 262-272.

Kausle R, D. H., \& KamichofF, N. C. Free recall of homographs and their primary associates. Journal of Verbal Learning and Verbal Behavior, 1970, 9, 79-83.

Kausler, D. H., \& Kollasch, S. F. Word associations to homographs. Journal of Verbal Learning and Verbal Behavior, $1970,9,444-449$.

Light, L. L., \& Carter-Sobell, L. Effects of changed semantic context on recognition memory. Journal of Verbal Learning and Verbal Behavior, 1970, 9, 1-11.

Macmillan dictionary for children. New York: Macmillan, 1975. Muller, J. H., Brown, S. C., \& Kausler, D. H. Pairedassociate transfer with homographic stimuli. Journal of Experimental Psychology: Human Learning and Memory, 1975, 1, $167 \cdot 172$.
Rowe, E. J. Frequency judgements and recognition of homonyms. Journal of Verbal Learning and Verbal Behavior, 1973, 12, 440-447.

Skanes, G., \& Donderı, D. C. Stimulus set, response set, and word identification. Journal of Experimental Psychology, 1973, 99, 413-423.

Spreen, O., \& Schulz, R. W. Parameters of abstraction, meaningfulness, and pronunciability for 329 nouns. Journal of Verbal Learning and Verbal Behavior, 1966, 5, 459-468.

Whitfond, H. C. A dictionary of American homophones and homographs. New York: Teachers College Press, 1966.

Winograd, E., \& Conn, C. P. Evidence from recognition memory for specific encoding of unmodified homographs. Journal of Verbal Learning and Verbal Behavior, 1971, 10, 702-706.

(Received for publication January 13, 1980; accepted January 22,1980 .) 